\title{
EDITORIAL
}

\section{Arthritis Research \& Therapy evolves: online only from 2011}

\author{
lain HrynaszkiewiczZ ${ }^{1 *}$, Peter E Lipsky² and Ravinder N Maini²
}

\section{Introduction}

Arthritis Research \& Therapy continues to evolve. When Arthritis Research - Arthritis Research \& Therapy's title until 2003 - was conceived over 10 years ago, it was projected to be the first fully online open access journal in rheumatology. We successfully pioneered the open access model in rheumatology and soon established unique qualities (described below), and feel we have become a leader in the worldwide progress toward open access publishing.

The Internet has fundamentally changed publishing, and scholarly articles are now primarily accessed electronically. In 2005 European online sales in scientific, technical and medical publishing were greater than print sales for the first time [1], and in 2009 it was reported that nearly $10 \%$ of all scientific, technical and medical journals were published under the open access model [2]. Indeed, the Directory of Open Access Journals now indexes more than 5,000 journals [3].

In 2002 Arthritis Research made the innovative decision - later replicated in other leading journals - to publish research articles in full online only, with the print journal being a compendium of what has been recently published online. Now that we have established ourselves as a leading rheumatology journal, it is time to move to the next stage of our evolution and become a fully online journal.

We believe that the rheumatology world has become much more comfortable with electronic communication in the past decade, and we feel that a fully electronic format will enhance the functionality and purposefulness of the journal and will provide a better service to readers and authors. The success of online, open access, publications has long assuaged any theoretical concerns about the permanency and impact of online-only articles. Since we can no longer identify a necessity for a regularly

*Correspondence: editorial@arthritis-research.com

'Associate Journal Publisher, BioMed Central, 236 Gray's Inn Road, London WC1X $8 \mathrm{HL}$, UK

Full list of author information is available at the end of the article printed journal we can no longer justify its adverse environmental impact, of which there is increasing awareness. The last regular printed issue of Arthritis Research \& Therapy will therefore be published at the end of 2010 and distributed in January 2011.

\section{Recent developments}

Regular readers and authors of Arthritis Research \& Therapy will have noticed that in January 2010 we implemented a complete redesign of the layout of our article PDFs - both the print and online versions. Improvements were made based on feedback from authors and readers; the new look provides a cleaner and more modern appearance, and helps better prepare us to move to online-only publication. For example, in recognition of that fact that articles are predominantly accessed online on an individual-article basis, you will find Arthritis Research \& Therapy's logo clearly on the first page of every online PDF.

As we move to an online-only format we will still publish a single highlights issue every year, which will include the most frequently downloaded and cited articles, for distribution at major rheumatology meetings. We believe this strategy will help us to reach a wide readership and recognizes that some functions remain for printed material when there is a clear and environmentally justifiable need, such as increasing our visibility to relevant audiences at academic conferences.

\section{Future developments}

Liberating ourselves of print for all of the journal's articles will free us from traditional limitations, and will bring other benefits. For example, it will enable us to publish even more cutting-edge, value-added content such as reviews and editorials quickly, which we believe are appropriate for this rapidly changing field. Authors of full-length, printed articles with illustrations and photographs will also benefit as they will no longer be faced with the potential costs of reproducing color figures for the print journal.

In the past 2 years we have demonstrated that special, online-only collections of articles in Arthritis Research \& Therapy can have high impact. In 2008/09 we published 
our 10th anniversary collection of 38 comprehensive reviews, 'The Scientific Basis of Rheumatology: A Decade of Progress', which was published in four parts alongside regular review articles and is only available online [4]. It has been accessed more than 180,000 times since the first articles were published in October 2008. We plan to provide more coverage of broad-interest article collections in the coming months, facilitated by the evolution of Arthritis Research \& Therapy into a fully online journal, which includes an exciting new series of reviews that will provide a comprehensive overview of the immunologic basis of rheumatic diseases.

Arthritis Research \& Therapy has always aimed to take full advantage of the panoply of benefits of online publishing. Articles in the journal can be shared via social media such as the currently popular Facebook and Twitter, and can be incorporated into personalized online literature services such as Mendeley. Our authors have also, for example, long been able to incorporate video, datasets and other online supplementary material with their articles. Increasing use of the Internet to augment printed matter is a trend across journals in general, although some online features, such as post-publication critique, have yet to become well established [5]. By publishing the journal online only, however, we increase our flexibility and ability to respond to our readers' and authors' needs, and we will soon be communicating with our readers to assess the demand for other additional functionality in the journal, such as podcasts and weblogs, within the rheumatology community. These and other exciting potential developments will be further enabled by a complete redesign of the journal website, which we will be unveiling in the coming months.

We encourage our readers and authors to contact the journal (editorial@arthritis-research.com) with suggestions for what more we can offer the rheumatology community, as we usher in the latest phase of the evolution of Arthritis Research \& Therapy (http://arthritis-research.com).

\section{Author details}

'Associate Journal Publisher, BioMed Central, 236 Gray's Inn Road, London WC1X 8HL, UK. '2Editor-in-Chief, Arthritis Research \& Therapy, 236 Gray's Inn Road, London WC1X 8HL, UK.

Published: 25 August 2010

\section{References}

1. Mort D: Online passes print in STM publishing. Research Information 2006 [http://www.researchinformation.info/riaugsep06analysis.html]

2. Pollock D: An Open Access Primer - Market Size and Trends. Outsell Inc.; 2009 [http://www.outsellinc.com/store/products/873].

3. DOAJ reaches new milestones - May 10, 2010 [http://www.doaj.org/doaj?func=loadTempl\&templ=100510]

4. Hrynaszkiewicz I, Lipsky PE, Maini RN: Introducing Arthritis Research \& Therapy's 10th anniversary issue, 'The Scientific Basis of Rheumatology: A Decade of Progress' [editorial]. Arthritis Res Ther 2008, 10:119.

5. Schriger DL, Merchant, R, Chehrazi A, Altman DG: Use of the Internet by print medical journals in 2003-2009: a longitudinal observational study [abstract]. [http://www.ama-assn.org/public/peer/abstracts_2009.html\#51]

doi:10.1186/ar3109

Cite this article as: Hrynaszkiewicz I, et al:: Arthritis Research \& Therapy evolves: online only from 2011. Arthritis Research \& Therapy 2010, 12:137. 